\title{
Pituitary adenoma and meningioma simulating a single selar and
}

\section{paraseal injury}

\section{Carlos Pérez-López ${ }^{1 *}$, Alexis J Palpán Flores ${ }^{1}$, Sharona Azriel ${ }^{2}$, Víctor Rodríguez-Domínguez ${ }^{1}$, Remedios Frutos ${ }^{3}$, Cristina Álvarez-Escolá ${ }^{4}$ and Alberto Isla ${ }^{1}$}

\author{
1'Department of Neurosurgery, Hospital Universitario La Paz, Madrid, Spain \\ ${ }^{2}$ Department of Endocrinology, Hospital Infanta Sofía, San Sebastián de los Reyes, Spain \\ ${ }^{3}$ Department of Neuroradiology, Hospital Universitario La Paz, Madrid, Spain \\ ${ }^{4}$ Department of Endocrinology, Hospital Universitario La Paz, Madrid, Spain
}

\section{Objective}

To analyze the importance of including axial cuts in studies of any brain region, including the selar. Remember the possibility of the existence of two different tumors simultaneously, in the same anatomical region.

\section{Methods}

We present the case of a 57-year-old woman with the clinical and analytical diagnosis of acromegaly. The pituitary MRI did not include axial cuts, but it clearly showed after the interpretation of the radiologist, endocrinologist and neurosurgeon, a right selar and parasellar lesion, which medially displaced the pituitary gland, with a size of $40 \mathrm{x}$ $32 \times 28 \mathrm{~mm}$, which laterally surpassed the carotid artery and reached the Gasser ganglion region (Figure 1). With the diagnosis of pituitary macroadenoma producing GH, Knosp grade III, surgery was indicated.

\section{Results}

An expanded endonasal endoscopic approach was carried

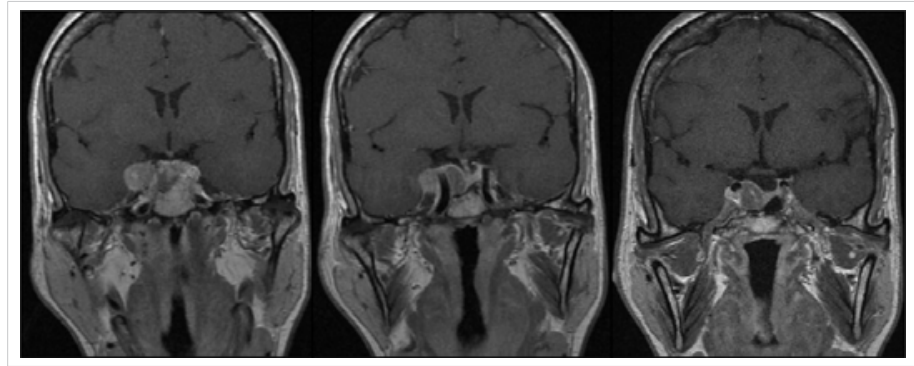

Figure 1: Preoperative MRI showing a sellar mass with a significant portion lateral to internal carotid artery (Knosp 3). The preoperative diagnosis was pituitary macroadenoma GH secreting.
More Information

*Address for Correspondence: Carlos Pérez-López, Department of Neurosurgery, Hospital Universitario La Paz, Madrid, Spain, Email: cperezhulp@yahoo.es

Submitted: October 15, 2020

Approved: August 23, 2021

Published: August 24, 2021

How to cite this article: Pérez-López C, Flores AJP, Azriel S, Rodríguez-Domínguez V, Frutos $\mathrm{R}$, et al. Pituitary adenoma and meningioma simulating a single selar and paraseal injury. J Neurosci Neurol Disord. 2021; 5: 087-088.

DOI: 10.29328/journal.jnnd.1001056

Copyright: ( 2021 Pérez-López C, et al. This is an open access article distributed under the Creative Commons Attribution License, which permits unrestricted use, distribution, and reproduction in any medium, provided the original work is properly cited.

\section{D) Check for updates}

OPEN ACCESS

out, remaining in the posterolateral area to the cavernous sinus. The pathological anatomy report confirmed that it was a GH-producing pituitary adenoma. In the postoperative period the patient had transient hypoesthesia in the territory of the first branches of the trigeminal nerve, and subsequent analyses showed, surprisingly, a cure of acromegaly. In control MRI, including this time axial cuts, an extraaxial lesion of $20 \times 16 \times$ $12 \mathrm{~mm}$ appeared, compatible with meningioma, centered on the ipsilateral petroclival ligament to the adenoma (Figure 2). Although this mass wasn't biopsed, with the diagnosis of suspected meningioma, it would later receive radiosurgery with volumetric stability after 8 years of follow-up [1-11].

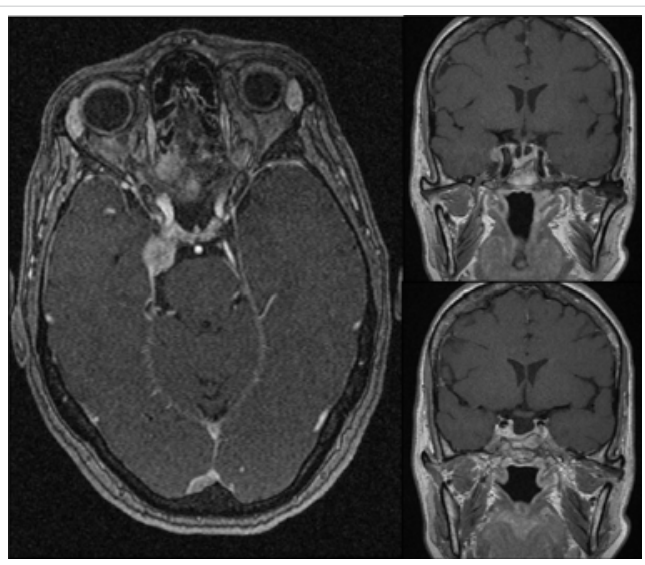

Figure 2: Postoperative MRI. In axial image a lesión attached to petroclinoidal ligament is observed. The postoperative diagnosis was pituitary adenoma and meningioma. 


\section{Conclusion}

It is essential to include axial cuts in all studies of the sellar region, since there are lesions that are difficult to characterize with only sagittal and coronal images. In this case, a cure of acromegaly after not completely removing what we thought was just adenoma, this proves it to us. In addition, it is a rare case of synchronous onset of meningioma and pituitary adenoma, which because it was intimately related, we were not able to diagnose correctly.

\section{References}

1. de Vries F, Lobatto DJ, Zamanipoor Najafabadi AH, Kleijwegt MC Verstegen MJT, et al. Unexpected concomitant pituitary adenoma and suprasellar meningioma: a case report and review of the literature. $\mathrm{Br}$ J Neurosurg. 2019; 1-5.

PubMed: https://pubmed.ncbi.nlm.nih.gov/31204511/

2. Zhao $\mathrm{Y}$, Zhang $\mathrm{H}$, Lian $\mathrm{W}$, Xing $\mathrm{B}$, Feng $\mathrm{M}$, et al. Collision tumours composed of meningioma and growth hormone-secreting pituitary adenoma in the sellar region: case reports and a literature review. Medicine. 2017; 96: e9139.

PubMed: https://pubmed.ncbi.nlm.nih.gov/29390316/

3. Ruiz-Juretschke F, Iza B, Scola-Pliego E, Poletti D, Salinero E. Coincidental pituitary adenoma and planum sphenoidale meningioma mimicking a single tumour. Endocrinol Nutr. 2015; 62: 292-294. PubMed: https://pubmed.ncbi.nlm.nih.gov/25963837/

4. Abs R, Parizel PM, Willems PJ, Van de Kelft E, Verlooy J, et al. The association of meningioma and pituitary adenoma: report of seven cases and review of the literature. Eur Neurol. 1993; 33: 416-422. PubMed: https://pubmed.ncbi.nlm.nih.gov/8307062/

5. Cannavò S, Curtò L, Fazio R, Paterniti S, Blandino A, et al. Coexistence of growth hormone-secreting pituitary adenoma and intracranial meningioma: a case report and review of the literature. J Endocrinol Invest. 1993; 16: 703-708.

PubMed: https://pubmed.ncbi.nlm.nih.gov/8282967/

6. Curto L, Squadrito S, Almoto B, Longo M, Granata F, et al. MRI finding of simultaneous coexistence of growth hormone-secreting pituitary adenoma with intracranial meningioma and carotid artery aneurysms: report of a case. Pituitary. 2007; 10: 299-205.

PubMed: https://pubmed.ncbi.nlm.nih.gov/17334927/

7. Karsy M, Sonnen J, Couldwell WT. Coincident pituitary adenoma and sellar meningioma. Acta Neurochirurgica 2015; 157: 231-233. PubMed: https://pubmed.ncbi.nlm.nih.gov/25416401/

8. Prevedello DM, Thomas A, Gardner P, Snyderman CH, Carrau RL, et al. Endoscopic endonasal resection of a synchronous pituitary adenoma and a tuberculum sellae meningioma: technical case report. Neurosurgery. 2007; 60: E401.

PubMed: https://pubmed.ncbi.nlm.nih.gov/17415151/

9. Yamada K, Hatayama T, Ohta M, Sakoda K, Uozumi T. Coincidental pituitary adenoma and parasellar meningioma: case report. Neurosurgery 1986; 19: 267-270.

PubMed: https://pubmed.ncbi.nlm.nih.gov/3748357/

10. Yu-Jen Lu CCC, Shih-Ming Jung KCW. Synchronous pituitary adenoma and tuberculum sellae meningioma. J Chinese Oncol Soc. 2008; 24: 269-274.

11. Zentner J, Gilsbach J. Pituitary adenoma and meningioma in the same patient. Report of three cases. Eur Arch Psychiatry Neurol Sci. 1989; 238: 144-148.

PubMed: https://pubmed.ncbi.nlm.nih.gov/2721531/ 